\title{
Index of Molecules
}

for materials (i.e., olivine, diamond, $\mathrm{CaO} . . .$.$) , see: Subject Index$

ammonia $\left(\mathrm{NH}_{3}\right) \quad 245,267,268,270$, 272,387

anthracene $\left(C_{14} H_{10}\right) \quad 187,188$

azulene $\left(\mathrm{C}_{10} \mathrm{H}_{8}\right) \quad 192,159,160,162,168$,

benz-perylene $\left(\mathrm{C}_{22} \mathrm{H}_{12}\right) \quad 186$

benzene $\left(\mathrm{C}_{6} \mathrm{H}_{6}\right) \quad 162,169,192,209$

carbon dioxide $\left(\mathrm{CO}_{2}\right)$ 244, 251, 424

carbon monosulfide $(C S) \quad\{24$

carbon monoxide $(C O)$ 243, 244, 247$251,265,270,271,295,346-349$, $399,423,424,455$

carbonyl sulfide (OCS) 251, $\$ 46,949$, 415,424

chrysene $\left(C_{18} H_{12}\right) \quad 199,151,202$

circumbiphenyl, circobiphenyl, circumdiphenyl $\left(\mathrm{C}_{38} \mathrm{H}_{16}\right) \quad 195,186$

cyano radical $(C N) \quad 265,428$

coronene $\left(C_{24} H_{12}\right) \quad 199,195,151,162$, 165, 176-178, 186, 207, 209, 213, 214, 322

cyanopolyynes $\left(H C_{2 n+1} N\right) \quad 264$

dicoronene $\left(\mathrm{C}_{48} \mathrm{H}_{20}\right) \quad 195$

formaldehyde $\left(\mathrm{H}_{2} \mathrm{CO}\right) \quad 349$

hexabenzocoronene $\left(\mathrm{C}_{42} \mathrm{H}_{18}\right) \quad 186$

hydrogen cyanide $(H C N) \quad 272,387$

hydrogen sulfide $\left(\mathrm{H}_{2} \mathrm{~S}\right) \quad \$ \$ 99$

methane $\left(\mathrm{CH}_{4}\right) \quad 188,267,268,424$

methanol $\left(\mathrm{CH}_{3} \mathrm{OH}\right) \quad 245,249,251,264$, $265,272,849$

naphthalene $\left(C_{10} H_{8}\right) \quad 159,160,162,167$ 169

ovalene $\left(\mathrm{C}_{32} \mathrm{H}_{14}\right) \quad 195,162,186,219$

oxygen, molecular $\left(\mathrm{O}_{2}\right) \quad 428$

oxygen, atomic $\{2 S$

pentacene $\left(\mathrm{C}_{22} \mathrm{H}_{14}\right) \quad 162$

perylene $\left(\mathrm{C}_{20} \mathrm{H}_{12}\right) \quad 186,187,200$

pyrene $\left(\mathrm{C}_{16} \mathrm{H}_{10}\right) \quad 199$

sulfur, atomic $\$ 21$

sulfur, diatomic $\left(S_{2}\right) \quad\{24$ water $\left(\mathrm{H}_{2} \mathrm{O}\right) \quad 245,249,251,267,268$, $272,349,407,422-424$ 